\title{
Optimal Planar Orthogonal Skyline Counting Queries*
}

\author{
Gerth Stølting Brodal ${ }^{\dagger} \quad$ Kasper Green Larsen*
}

Arpil 24, 2014

\begin{abstract}
The skyline of a set of points in the plane is the subset of maximal points, where a point $(x, y)$ is maximal if no other point $\left(x^{\prime}, y^{\prime}\right)$ satisfies $x^{\prime} \geq x$ and $y^{\prime} \geq y$. We consider the problem of preprocessing a set $P$ of $n$ points into a space efficient static data structure supporting orthogonal skyline counting queries, i.e. given a query rectangle $R$ to report the size of the skyline of $P \cap R$. We present a data structure for storing $n$ points with integer coordinates having query time $O(\lg n / \lg \lg n)$ and space usage $O(n)$ words. The model of computation is a unit cost RAM with logarithmic word size. We prove that these bounds are the best possible by presenting a matching lower bound in the cell probe model with logarithmic word size: Space usage $n \lg ^{O(1)} n$ implies worst case query time $\Omega(\lg n / \lg \lg n)$.
\end{abstract}

\section{Introduction}

In this paper we consider orthogonal range skyline queries for a set of points in the plane. A point $(x, y) \in$ $\mathbb{R}^{2}$ dominates a point $\left(x^{\prime}, y^{\prime}\right)$ if and only if $x^{\prime} \leq x$ and $y^{\prime} \leq y$. For a set of points $P$, a point $p \in P$ is maximal if no other point in $P$ dominates $p$, and the skyline of $P$, Skyline $(P)$, is the subset of maximal points in $P$.

We consider the problem of preprocessing a set $P$ of $n$ points in the plane with integer coordinates into a data structure to support orthogonal range skyline counting queries: Given an axis-aligned query rectangle $R=\left[x_{1}, x_{2}\right] \times\left[y_{1}, y_{2}\right]$ to report the size of the skyline of the subset of the points from $P$ contained in $R$, i.e. report $\mid$ Skyline $(P \cap R) \mid$. The main results of this paper are matching upper and lower bounds for data structures supporting such queries, thus completely settling the problem. Our model of computation is the standard unit cost RAM with logarithmic word size.

\subsection{Previous Work}

Orthogonal range searching is one of the most fundamental and well-studied topics in computational geometry, see e.g. [4] for an extensive list of previous results. For orthogonal range queries in the plane, with integer coordinates in $[n] \times[n]=\{0, \ldots, n-1\} \times\{0, \ldots, n-1\}$, the main results are the following: For the orthogonal range counting problem, i.e. queries report the total number of input points inside a query rectangle, optimal $O(\lg n / \lg \lg n)$ query time using $O(n)$ space was achieved in [8]. Optimality was shown

\footnotetext{
${ }^{*}$ Full version of paper appearing in the proceedings of the 14th Scandinavian Symposium and Workshops on Algorithm Theory, 2014.

${ }^{\dagger}$ MADALGO, Center for Massive Data Algorithmics, a Center of the Danish National Research Foundation (grant DNRF84). Department of Computer Science, Aarhus University. \{gerth, larsen\}@ cs . au.dk.
} 
Table 1: Previous and new results for skyline counting queries.

\begin{tabular}{ccl} 
Space (words) & Query time & Reference \\
\hline$n \frac{\lg ^{2} n}{\lg \lg n}$ & $\frac{\lg ^{3 / 2} n}{\lg \lg n}$ & {$[5]$} \\
$n \lg n$ & $\lg n$ & {$[9]$} \\
$n \frac{\lg n}{\lg \lg n}$ & $\frac{\lg n}{\lg \lg n}$ & {$[6]$} \\
$n$ & $\frac{\lg n}{\lg \lg n}$ & New \\
\hline
\end{tabular}

Table 2: Previous and new results for skyline reporting queries.

\begin{tabular}{ccl} 
Space (words) & Query time & Reference \\
\hline$n \lg n$ & $\lg ^{2} n+k$ & {$[3]$ (dynamic) } \\
$n \lg n$ & $\lg n+k$ & {$[10,9]$} \\
$n \frac{\lg n}{\lg \lg n}$ & $\frac{\lg n}{\lg \lg n}+k$ & {$[5]$} \\
$n \lg ^{\varepsilon} n$ & $(k+1) \lg \lg n$ & {$[11]$} \\
$n \lg ^{\varepsilon} n$ & $\frac{\lg n}{\lg n}+k$ & New \\
$n \lg \lg n$ & $(k+1)(\lg \lg n)^{2}$ & {$[11]$} \\
$n \lg \lg n$ & $\lg n$ \\
$\lg \lg n+k \lg \lg n$ & New \\
$n$ & $(k+1) \lg ^{\varepsilon} n$ & {$[11]$} \\
\hline
\end{tabular}

in [13], where it was proved that space $n \lg ^{O(1)} n$ implies query time $\Omega(\lg n / \lg \lg n)$ for range counting queries.

For range reporting queries it is known that space $n \lg ^{O(1)} n$ implies query time $\Omega(\lg \lg n+k)$, where $k$ is the number of points reported within the query range [14]. The best upper bounds known for range reporting are: Optimal space $O(n)$ and query time $O\left((k+1) \lg ^{\varepsilon} n\right)$ [4], and optimal query time $O(\lg \lg n+k)$ with space $O\left(n \lg ^{\varepsilon} n\right)$ [1]. In both cases $\varepsilon>0$ is an arbitrarily small constant.

Orthogonal Range Skyline Queries. Orthogonal range skyline counting queries were first consider in [5], where a data structure was presented with space usage $O\left(n \lg ^{2} n / \lg \lg n\right)$ and query time $O\left(\lg ^{3 / 2} n / \lg \lg n\right)$. This was subsequently improved to $O(n \lg n)$ space and $O(\lg n)$ query time [9]. Finally, a data structure achieving an even faster query time of $O(\lg n / \lg \lg n)$ was presented, however the space usage of that solution was a prohibitive $O\left(n \lg ^{3} n / \lg \lg n\right)$ [6]. Thus to date, no linear space solution exists with a non-trivial query time. Also, from a lower bound perspective, it is not known whether the problem is easier or harder than the standard range counting problem.

For orthogonal skyline reporting queries, the best bound is $O(n \lg n / \lg \lg n)$ space with query time $O(\lg n / \lg \lg n+k)$ [5], where $k$ is the size of the reported skyline. Note that an $\Omega(\lg \lg n)$ search term is needed for skyline range reporting since the $\Omega(\lg \lg n)$ lower bound for standard range reporting was proved even for the case of determining whether the query rectangle is empty [14].

In [11] solutions for the sorted range reporting problem were presented, i.e. the problem of reporting the $k$ leftmost points within a query rectangle in sorted order of increasing $x$-coordinate. With space $O(n)$, $O(n \lg \lg n)$ and $O\left(n \lg ^{\varepsilon} n\right)$, respectively, query times $O\left((k+1) \lg ^{\varepsilon} n\right), O\left((k+1)(\lg \lg n)^{2}\right)$, and $O(k+$ $\lg \lg n)$ were achieved, respectively. The structures of [11] support finding the rightmost (skyline) point in a query range $(k=1)$. By recursing on the rectangle above the reported point one immediately get the bounds for skyline reporting listed in Table 2, where only the linear space solution achieves query times matching those of general orthogonal range reporting.

Previous results for skyline queries are summarized in Tables 1 and 2.

\subsection{Our Results}

In Section 3 we present a linear space data structure supporting orthogonal range skyline counting queries in $O(\lg n / \lg \lg n)$ time, thus for the first time achieving linear space and improving over all previous tradeoffs. 
In Section 2 we show that this is the best possible by proving a matching lower bound. More specifically, we prove a lower bound stating that the query time $t$ must satisfy $t=\Omega(\lg n / \lg (S w / n))$. Here $S \geq n$ is the space usage in number of words and $w=\Omega(\lg n)$ is the word size in bits. For $\left.w=\lg ^{O(1)} n\right)$ and $S=n \lg ^{O(1)} n$, this bound becomes $t=\Omega(\lg n / \lg \lg n)$. The lower bound is proved in the cell probe model of Yao [18], which is more powerful than the unit cost RAM and hence the lower bound also applies to RAM data structures.

As a side result, we in Section 4 show how to modify our counting data structure to support reporting queries. Our reporting data structure has query time $O(\lg n / \lg \lg n+k)$ and space usage $O\left(n \lg ^{\varepsilon} n\right)$. The best previous reporting structure with a linear term in $k$ has $O(\lg n / \lg \lg n+k)$ query time but $O(n \lg n / \lg \lg n)$ space [5]. The reporting structure can also be modified to achieve $O(\lg n / \lg \lg n+$ $k \lg \lg n)$ query time and $O(n \lg \lg n)$ space. See Table 2 for a comparison to previous results.

Our lower bound follows from a reduction of reachability in butterfly graphs to two-sided skyline counting queries, extending reductions by Pătraşcu [12] for two-dimensional rectangle stabbing and range counting queries. Our upper bounds are achieved by constructing a balanced search tree of degree $\Theta\left(\lg ^{\varepsilon} n\right)$ over the points sorted by $x$-coordinate. At each internal node we store several space efficient rank-select data structures storing the points in the subtrees sorted by rank-reduced $y$-coordinates. Using a constant number of global tables, queries only need to spend $O(1)$ time at each level of the tree.

\subsection{Preliminaries}

Coordinates. If the coordinates of the input and query points are not restricted to $[n] \times[n]$, but can be arbitrary integers that fit into a machine word, then we can map the coordinates to the range $[n]$ by using the RAM dictionary from [2], which can support predecessor queries on the lexicographical orderings of the points in time $O(\sqrt{\lg n / \lg \lg n})$ using $O(n)$ space. This is less than the $O(\lg n / \lg \lg n)$ query time we are aiming for.

Succinct Data Structures. In our solutions, we make extensive use of the following results from succinct data structures.

Lemma 1 ([15]) A vector $X[1 . . s]$ of s zero-one values, with $t$ values equal to one, can be stored in a data structure of size $O(t(1+\lg s / t))$ bits supporting rank and select queries in $O(1)$ time. A $\operatorname{rank}(i)$ query returns the number of ones in $X[1 . . i]$, provided $X[i]=1$, whereas a select $(i)$ query returns the position of the $i$ 'th one in $X$.

Lemma 2 ([16]) Let $X[1 . . s]$ be a vector of s non-negative integers with total sum $t$. There exists a data structure of size $O(s \lg (2+t / s))$ bits, supporting the lookup of $X[i]$ and the prefix sum $\sum_{j=1}^{i} X[j]$ in $O(1)$ time, for $i=1, \ldots, s$.

Lemma $3([17,7])$ Let $X[1 . . s]$ be a vector of integers. There exists a data structure of size $O(s)$ bits supporting range-maximum-queries in $O(1)$ time, i.e. given $i$ and $j, 1 \leq i \leq j \leq s$, reports the index $k$, $i \leq k \leq j$, such that $X[k]=\max (X[i . . j])$. Queries only access this data structure, i.e. the vector $X$ is not stored.

\section{Lower Bound}

That an orthogonal range skyline counting data structure requires space $\Omega(n \lg n)$ bits, follows immediately since each of the $n$ ! different input point sets of size $n$, where points have distinct $x$ - and $y$-coordinates 
from $[n]$, can be reconstructed using query rectangles considering each possible point in $[n]^{2}$ independently, i.e. the space usage is at least $\left\lceil\lg _{2}(n !)\right\rceil=\Omega(n \lg n)$ bits.

In the remainder of this section, we prove that any data structure using $S \geq n$ words of space must have query time $t=\Omega(\lg n / \lg (S w / n))$, where $w=\Omega(\lg n)$ denotes the word size in bits. In particular for $w=\lg ^{O(1)} n$, this implies that any data structure using $n \lg ^{O(1)} n$ space must have query time $t=$ $\Omega(\lg n / \lg \lg n)$, showing that our data structure from Section 3 is optimal. Our lower bound holds even for data structures only supporting skyline counting queries inside 2 -sided rectangles, i.e. query rectangles of the form $(-\infty, x] \times(-\infty, y]$. The lower bound is proved in the cell probe model of Yao [18] with word size $w=\Omega(\lg n)$. Since we derive our lower bound by reduction, we will not spend time on introducing the cell probe model, but merely note that lower bounds proved in this model applies to data structures developed in the unit cost RAM model. See e.g. [13] for a brief description of the cell probe model.

Reachability in the Butterfly Graph. We prove our lower bound by reduction from the problem known as reachability oracles in the butterfly graph [12]. A butterfly graph of degree $B$ and depth $d$ is a directed graph with $d+1$ layers, each having $B^{d}$ nodes ordered from left to right (see Figure 1). The nodes at level 0 are the sources and the nodes at level $d$ are the sinks. Each node, except the sinks, has out-degree $B$, and each node, except the sources, has in-degree $B$.

If we number the nodes at each level with $0, \ldots, B^{d}-1$ from left to right and interpret each index $i \in\left[B^{d}\right]$ as a vector $v(i)=v(i)[d-1] \cdots v(i)[0] \in[B]^{d}$ (just write $i$ in base $B$ ), then the node at index $i$ at layer $k \in[d]$ has an out-going edge to each node $j$ at layer $k+1$ for which $v(j)$ and $v(i)$ differ only in the $k$ 'th coordinate. Here the 0 'th coordinate is the coordinate corresponding to the least significant digit when thinking of $v(i)$ and $v(j)$ as numbers written in base $B$ (again see Figure 1). Observe that there is precisely one directed path between each source-sink pair. For the $s$ 'th source and the $t$ 'th sink, this path corresponds to "morphing" one digit of $v(s)$ into the corresponding digit in $v(t)$ for each layer traversed in the butterfly graph.

The input to the problem of reachability oracles in the butterfly graph, with degree $B$ and depth $d$, is a subset of the edges of the butterfly graph, i.e. we are given a subgraph $G$ of the butterfly as input. A query is specified by a source-sink pair $(s, t)$ and the goal is to return whether there exists a directed path from the given source $t$ to the given sink $t$ in $G$.

Pătraşcu proved the following lower bound for reachability oracles:

Theorem 1 (Pătraşcu [12], Section 5) Any cell probe data structure answering reachability queries in subgraphs of the butterfly graph with degree $B$ and depth $d$, having space usage $S$ words of $w$ bits, must have query time $t=\Omega(d)$, provided $B=\Omega\left(w^{2}\right)$ and $\lg B=\Omega(\lg S d / N)$. Here $N$ denotes the number of non-sink nodes in the butterfly graph.

We derive our lower bound by showing that any cell probe data structure for skyline range counting can be used to answer reachability queries in subgraphs of the butterfly graph for any degree $B$ and depth $d$.

Edges to 2-d Rectangles. Consider the butterfly graph with degree $B$ and depth $d$. The first step of our reduction is inspired by the reduction Pătraşcu used to obtain a lower bound for 2-d rectangle stabbing: Consider an edge of the butterfly graph, leaving the $i$ 'th node at layer $k \in[d]$ and entering the $j$ 'th node in layer $k+1$. We denote this edge $e_{k}(i, j)$. The source-sink pairs $(s, t)$ that are connected through $e_{k}(i, j)$ are those for which:

1. The source has an index $s$ satisfying $v(s)[h]=v(i)[h]$ for $h \geq k$, i.e. $s$ and $i$ agree on the $d-k$ most significant digits when written in base $B$. 


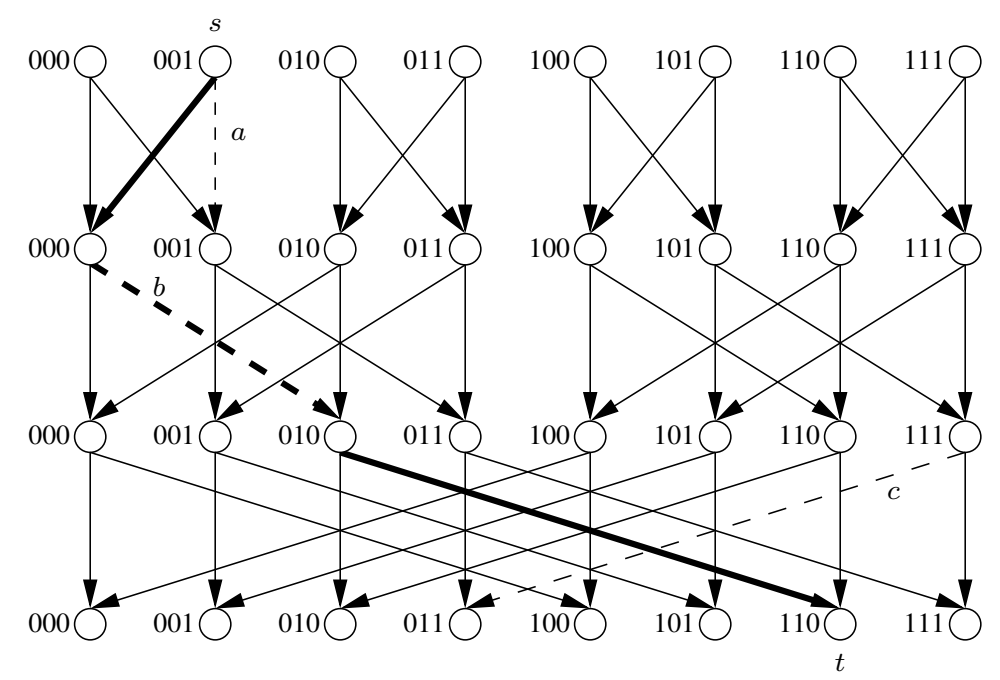

Figure 1: A butterfly with degree $B=2$ and depth $d=3$. The path shown in bold is the unique path from the source $s=001$ to the sink $t=110$. A concrete input to the reachability oracles in the butterfly graph problem consists of a subset of the edges of the butterfly. An example input is obtained by deleting the dashed edges labelled $a, b$ and $c$. For that input, there is no path from the source $s$ to the sink $t$ since the edge $b$ is not part of the input.

2. The sink has an index $t$ satisfying $v(t)[h]=v(j)[h]$ for $h \leq k+1$, i.e. $t$ and $j$ agree on the $k+1$ least significant digits when written in base $B$.

We now map each edge $e_{k}(i, j)$ of the butterfly graph to a rectangle in 2-d. For the edge $e_{k}(i, j)$, we create the rectangle $r_{k}(i, j)=\left[x_{1}, x_{2}\right] \times\left[y_{1}, y_{2}\right]$ where:

- $x_{1}=v(i)[d-1] v(i)[d-2] \cdots v(i)[k] 0 \cdots 0$ when written in base $B$,

- $x_{2}=v(i)[d-1] v(i)[d-2] \cdots v(i)[k](B-1) \cdots(B-1)$ when written in base $B$,

- $y_{1}=v(j)[0] v(j)[1] \cdots v(j)[k+1] 0 \cdots 0$ when written in base $B$, and

- $y_{2}=v(j)[0] v(j)[1] \cdots v(j)[k+1](B-1) \cdots(B-1)$ when written in base $B$.

The crucial observation is that for a source-sink pair, where the source is the $s$ 'th source and the sink is the $t$ 'th sink, the edges on the path from the source to the sink in the butterfly graph are precisely those edges $e_{k}(i, j)$ for which the corresponding rectangle $r_{k}(i, j)$ contains the point $\left(s, \operatorname{rev}_{B}(t)\right)$, where $\operatorname{rev}_{B}(t)$ is the number obtained by writing $t$ in base $B$ and then reversing the digits.

We now collect the set of rectangles $R$, containing each rectangle $r_{k}(i, j)$ corresponding to an edge of the butterfly graph. Given an input subgraph $G$, we mark all rectangles $r_{k}(i, j) \in R$ for which the corresponding edge $e_{k}(i, j)$ is also in $G$. It follows that there is a directed path from the $s$ 'th source to the $t^{\prime}$ th sink in the subgraph $G$ if and only if $\left(s, \operatorname{rev}_{B}(t)\right)$ is not contained in any unmarked rectangle in $R$.

Our goal is now to transform marked and unmarked rectangles to points, such that we can use a skyline counting data structure to determine whether a given point $\left(s, \operatorname{rev}_{B}(t)\right)$ is contained in an unmarked rectangle. Note that our reduction only works for the rectangle set $R$ obtained from the butterfly graph, and not for any set of rectangles, i.e. we could not have reduced from the general problem of 2-d rectangle stabbing. 
2-d Rectangles to Points. To avoid tedious details, we from this point on allow the input to skyline queries to have multiple points with the same $x$ - or $y$-coordinate (though not two points with both coordinates identical). This assumption can easily be removed, but it would only distract the reader from the main ideas of our reduction. We still use the definition that a point $(x, y)$ dominates a point $\left(x^{\prime}, y^{\prime}\right)$ if and only if $x^{\prime} \leq x$ and $y^{\prime} \leq y$.

The next step of the reduction is to map the rectangles $R$ to a set of points. For this, we first transform the coordinates slightly: For every rectangle $r_{k}(i, j) \in R$, having coordinates $\left[x_{1}, x_{2}\right] \times\left[y_{1}, y_{2}\right]$, we modify each of the coordinates in the following way:

$$
\begin{aligned}
& \text { - } x_{1} \leftarrow d x_{1}+(d-1-k), \\
& \text { - } x_{2} \leftarrow d x_{2}+d-1, \\
& \text { - } y_{1} \leftarrow d y_{1}+k \text {, and } \\
& \text { - } y_{2} \leftarrow d y_{2}+d-1 .
\end{aligned}
$$

The multiplication with $d$ essentially corresponds to expanding each point with integer coordinates to a $d \times d$ grid of points. The purpose of adding $k$ to $y_{1}$ and $(d-1-k)$ to $x_{1}$ is to ensure that, if two rectangles share a lower-left corner (only possible for two rectangles $r_{k}(i, j)$ and $r_{k^{\prime}}\left(i^{\prime}, j^{\prime}\right)$ where $k \neq k^{\prime}$ ), then those corners do not dominate each other in the transformed set of rectangles. We will see later that the particular placement of the points based on $k$ also plays a key role. We use $\pi:\left[B^{d}\right]^{4} \rightarrow\left[d B^{d}\right]^{4}$ to denote the above map. With this notation, the transformed set of rectangles is denoted $\pi(R)$ and each rectangle $r_{k}(i, j) \in R$ is mapped to $\pi\left(r_{k}(i, j)\right) \in \pi(R)$.

We now create the set of points $P^{\prime}$ containing the set of lower-left corner points for all rectangles $\pi\left(r_{k}(i, j)\right) \in \pi(R)$, i.e. for each $\pi\left(r_{k}(i, j)\right)=\left[x_{1}, x_{2}\right] \times\left[y_{1}, y_{2}\right]$, we add the point $\left(x_{1}, y_{1}\right)$ to $P^{\prime}$. See Figure 2 for an example. The set $P^{\prime}$ has the following crucial property:

Lemma 4 Let $(x, y)$ be a point with coordinates in $\left[B^{d}\right] \times\left[B^{d}\right]$. Then for the two-sided query rectangle $Q=(-\infty, d x+d-1] \times(-\infty, d y+d-1]$, it holds that $\operatorname{Skyline}\left(Q \cap P^{\prime}\right)$ contains precisely the points in $P^{\prime}$ corresponding to the lower-left corners of the rectangles $\pi\left(r_{k}(i, j)\right) \in \pi(R)$ for which $r_{k}(i, j)$ contains $(x, y)$.

Proof. First let $p=\left(x_{1}, y_{1}\right) \in P^{\prime}$ be the lower-left corner of a rectangle $\pi\left(r_{k}(i, j)\right)$ such that $r_{k}(i, j)$ contains the point $(x, y)$. We want to show that $p \in \operatorname{Skyline}\left(Q \cap P^{\prime}\right)$. Since $r_{k}(i, j)$ contains the point $(x, y)$, we have $x \geq\left\lfloor x_{1} / d\right\rfloor$ and $y \geq\left\lfloor y_{1} / d\right\rfloor$. From this, we get $d x+d-1 \geq d\left\lfloor x_{1} / d\right\rfloor+(d-1-k)=x_{1}$ and $d y+d-1 \geq d\left\lfloor y_{1} / d\right\rfloor+k=y_{1}$, i.e. $p$ is inside $Q$. Since $(x, y)$ is inside $r_{k}(i, j)$, we also have that $(d x+d-1, d y+d-1)$ is dominated by the upper-right corner of $\pi\left(r_{k}(i, j)\right)$, i.e. $(d x+d-1, d y+d-1)$ is inside $\pi\left(r_{k}(i, j)\right)$.

What remains to be shown is that no other point in $Q \cap P^{\prime}$ dominates $p$. For this, assume for contradiction that some point $p^{\prime}=\left(x_{1}^{\prime}, y_{1}^{\prime}\right) \in P^{\prime}$ is both in $Q$ and also dominates $p$. First, since $p^{\prime}$ is dominated by $(d x+d-1, d y+d-1)$ and also dominates $p$, we know that $p^{\prime}$ must be inside $\pi\left(r_{k}(i, j)\right)$. Now let $\pi\left(r_{k^{\prime}}\left(i^{\prime}, j^{\prime}\right)\right) \neq \pi\left(r_{k}(i, j)\right)$ be the rectangle in $\pi(R)$ from which $p^{\prime}$ was generated, i.e. $p^{\prime}$ is the lower-left corner of $\pi\left(r_{k^{\prime}}\left(i^{\prime}, j^{\prime}\right)\right)$. We have three cases:

1. First, if $k^{\prime}=k$ we immediately get a contradiction since the rectangles $\pi(R)_{k}=\left\{\pi\left(r_{k^{\prime}}\left(i^{\prime}, j^{\prime}\right)\right) \in\right.$ $\left.\pi(R) \mid k^{\prime}=k\right\}$ are pairwise disjoint and hence $p^{\prime}$ could not have been inside $\pi\left(r_{k}(i, j)\right)$. 


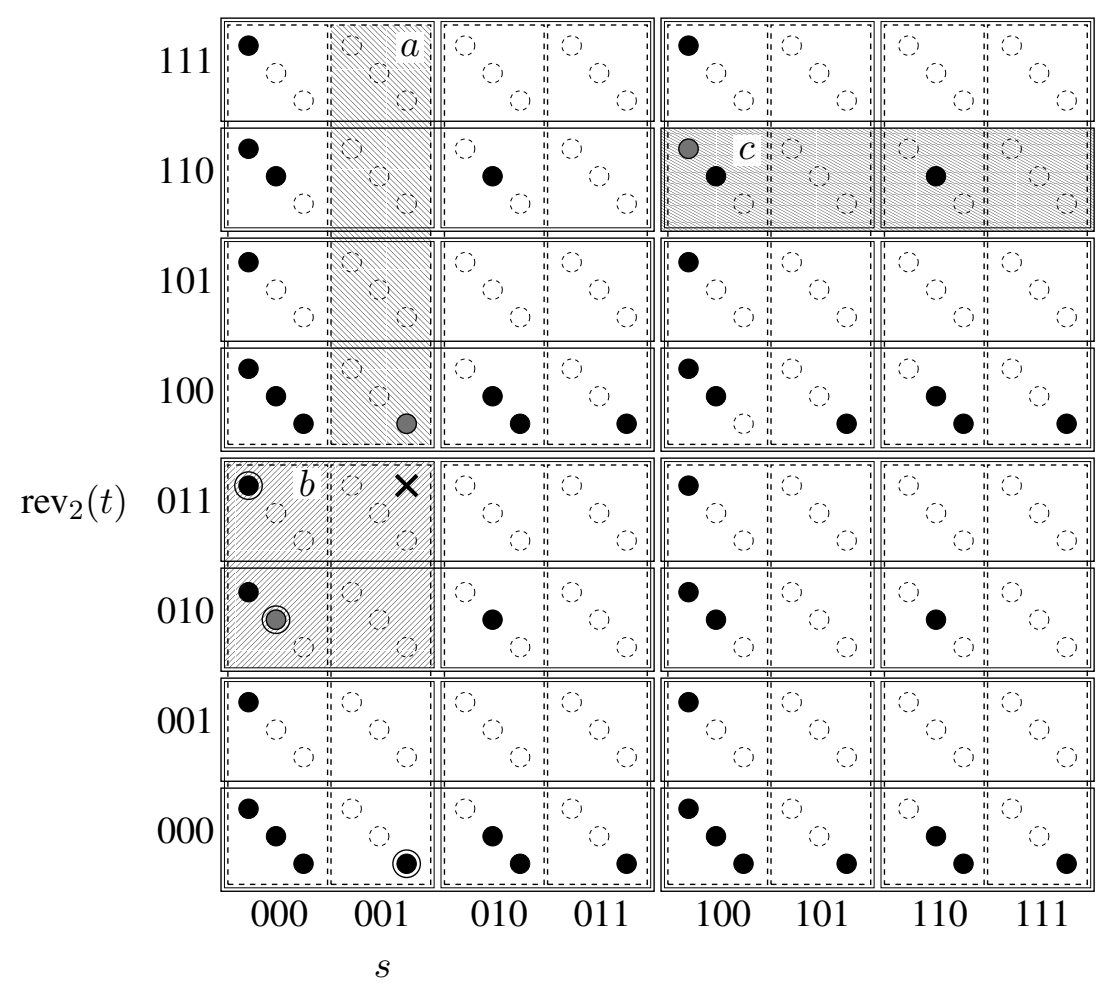

Figure 2: The butterfly with degree $B=2$ and depth $d=3$ from Figure 1 translated to a set of rectangles. The dashed rectangles correspond to the edges $a, b$ and $c$ from Figure 1. Every grid point is replaced by up to $d$ points placed on a diagonal. Each rectangle obtained from an edge of the butterfly graph produces one point on the diagonal corresponding to the rectangle's lower left corner. The points corresponding to the rectangles obtained from edges $a, b$ and $c$ are shown in gray. The query corresponding to the source $s=001$ and the sink $t=110$ in Figure 1 is translated to the two-sided skyline query rectangle with its upper right corner at the $\times$. The double circled points are the points on the skyline of the query range and these correspond exactly to the lower left corners of the rectangles containing the $\times$.

2. If $k^{\prime}<k$, we know that $\pi\left(r_{k^{\prime}}\left(i^{\prime}, j^{\prime}\right)\right)$ is shorter in $x$-direction and longer in $y$-direction than $\pi\left(r_{k}(i, j)\right)$. From our transformation, we know that $\left(y_{1} \bmod d\right)=k$ and $\left(y_{1}^{\prime} \bmod d\right)=k^{\prime}<k$. Thus since $p^{\prime}$ dominates $p$, we must have $\left\lfloor y_{1}^{\prime} / d\right\rfloor>\left\lfloor y_{1} / d\right\rfloor$. But these two values are precisely the $y$-coordinates of the lower-left corners of $r_{k}(i, j)$ and $r_{k^{\prime}}\left(i^{\prime}, j^{\prime}\right)$. By definition, we get:

$$
v\left(j^{\prime}\right)[0] v\left(j^{\prime}\right)[1] \cdots v\left(j^{\prime}\right)\left[k^{\prime}+1\right] 0 \cdots 0>v(j)[0] v(j)[1] \cdots v(j)[k+1] 0 \cdots 0 .
$$

Since $k^{\prime}<k$, this furthermore gives us

$$
v\left(j^{\prime}\right)[0] v\left(j^{\prime}\right)[1] \cdots v\left(j^{\prime}\right)\left[k^{\prime}+1\right]>v(j)[0] v(j)[1] \cdots v(j)\left[k^{\prime}+1\right] .
$$

From this it follows that

$$
v\left(j^{\prime}\right)[0] v\left(j^{\prime}\right)[1] \cdots v\left(j^{\prime}\right)\left[k^{\prime}+1\right] 0 \cdots 0>v(j)[0] v(j)[1] \cdots v(j)[k+1](B-1) \cdots(B-1),
$$

i.e. the lower-left corner of $r_{k^{\prime}}\left(i^{\prime}, j^{\prime}\right)$ is outside $r_{k}(i, j)$, which also implies that the lower-left corner of $\pi\left(r_{k^{\prime}}\left(i^{\prime}, j^{\prime}\right)\right)$ is outside $\pi\left(r_{k}(i, j)\right)$. That is, $p^{\prime}$ is outside $\pi\left(r_{k}(i, j)\right)$, which gives the contradiction. 


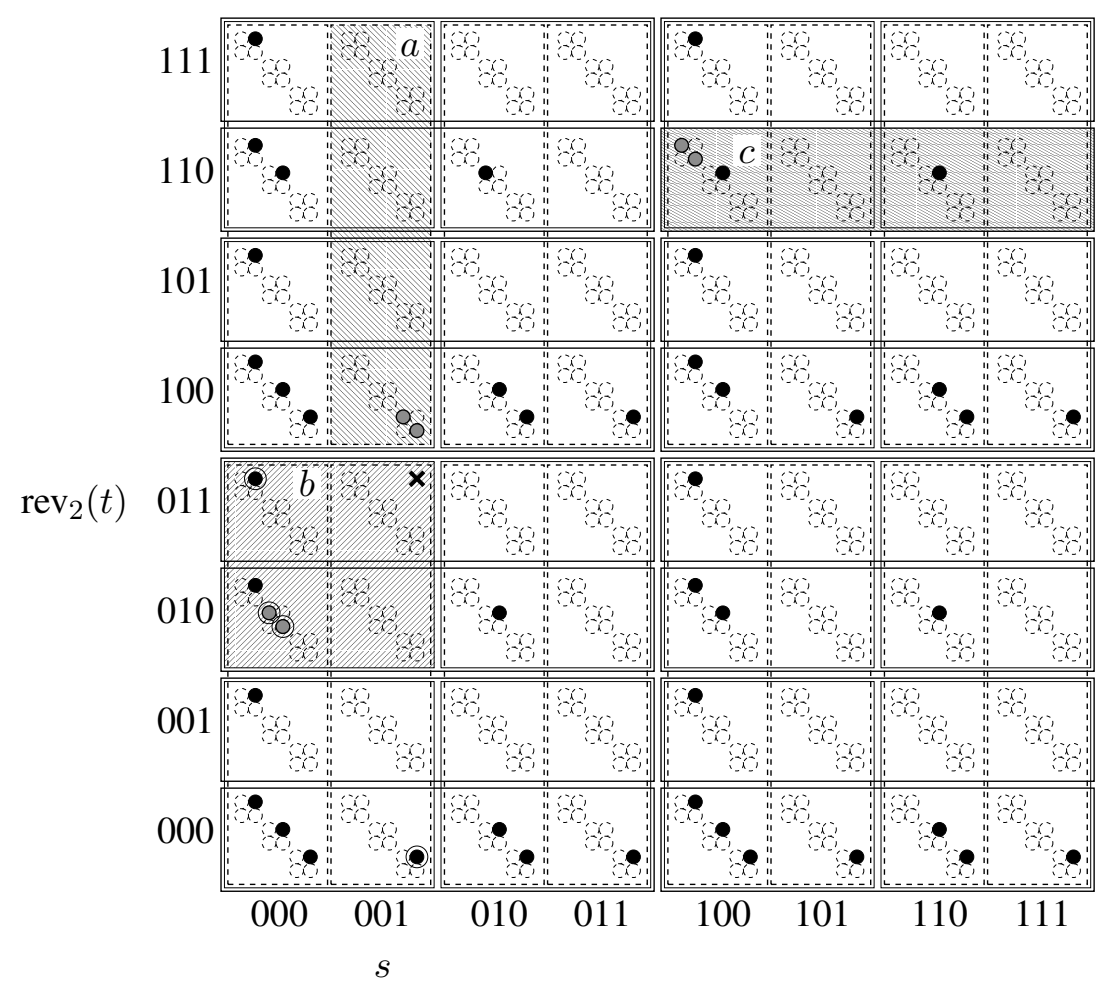

Figure 3: Points corresponding to unmarked rectangles are replaced by two points. The example from Figure 1 and Figure 2 has three unmarked rectangles, corresponding to edges $a, b$ and $c$ of the butterfly graph. As shown, these rectangles become two (gray) input points and marked rectangles are represented by only one input point. The upper right corner of the two-sided query rectangle corresponding to the source $s=001$ and sink $t=110$ in the previous examples is shown as a $\times$. The double circled points are the points on the skyline of the query range. As can be seen, the unmarked rectangle corresponding to the edge labelled $b$ contributes two points to the skyline of the query $\times$.

3. The case for $k^{\prime}>k$ is symmetric to the case $k^{\prime}<k$, just using the $x$-coordinates instead of the $y$-coordinates to derive the contradiction.

The last step of the proof is to show that no point $p=\left(x_{1}, y_{1}\right) \in P^{\prime}$ can be in $\operatorname{Skyline}\left(Q \cap P^{\prime}\right)$ but at the same time correspond to the lower-left corner of a rectangle $\pi\left(r_{k}(i, j)\right)$ where $r_{k}(i, j)$ does not contains the point $(x, y)$. First observe that $(d x+d-1, d y+d-1)$ is contained in precisely one rectangle $\pi\left(r_{k^{\prime}}\left(i^{\prime}, j^{\prime}\right)\right)$ for each value of $k^{\prime} \in[d]$. Now let $\pi\left(r_{k}\left(i^{\prime}, j^{\prime}\right)\right) \neq \pi\left(r_{k}(i, j)\right)$ be the rectangle containing $(d x+d-1, d y+d-1)$ amongst the rectangles $\pi(R)_{k}$. The lower-left corner of this rectangle is dominated by $(d x+d-1, d y+d-1)$ but also dominates $p$, hence $p$ is not in $\operatorname{Skyline}\left(Q \cap P^{\prime}\right)$.

Handling Marked and Unmarked Rectangles. The above steps are all independent of the concrete input subgraph $G$. As discussed, we need a way to determine whether a query point is contained in an unmarked rectangle or not. This step is now very simple in light of Lemma 4: First, multiply all coordinates of points in $P^{\prime}$ by 2 . This corresponds to expanding each point with integer coordinates into a $2 \times 2$ grid. Now for every point $p \in P^{\prime}$, if the rectangle $\pi\left(r_{k}(i, j)\right)$ from which $p$ was generated is marked, then we add 1 to both the $x$ - and $y$-coordinate of $p$, i.e. we move $p$ to the upper-right corner of the $2 \times 2$ grid in which it is 
placed. If $\pi\left(r_{k}(i, j)\right)$ is unmarked, we replace it by two points, one where we add 1 to the $x$-coordinate, and one where we add 1 to the $y$-coordinate, see Figure 3 . We denote the resulting set of points $P(G)$. It follows immediately that:

Corollary 1 Let $G$ be a subgraph of the butterfly graph with degree $B$ and depth $d$. Also, let $(x, y)$ be a point with coordinates in $\left[B^{d}\right] \times\left[B^{d}\right]$. Then for the two-sided query rectangle $Q=(-\infty, 2 d(x+1)-$ $1] \times(-\infty, 2 d(y+1)-1]$, it holds that Skyline $(Q \cap P(G))$ contains precisely one point from $P(G)$ for every marked rectangle in $R$ that contains $(x, y)$, two points from $P(G)$ for every unmarked rectangle in $R$ that contains $(x, y)$, and no other points, i.e. $\mid$ Skyline $(Q \cap P(G)) \mid-d$ equals the number of unmarked rectangles in $R$ which contains $(x, y)$.

Corollary 2 Let $G$ be a subgraph of the butterfly graph with degree $B$ and depth $d$. Let s be the index of a source and the index of a sink. Then the s'th source can reach the t'th sink in $G$ if and only if $\mid \operatorname{Skyline}(Q \cap$ $P(G)) \mid=d$ for the two-sided query rectangle $Q=(-\infty, 2 d(s+1)-1] \times\left(-\infty, 2 d\left(\operatorname{rev}_{B}(t)+1\right)-1\right]$.

Deriving the Lower Bound. The lower bound can be derived from Corollary 2 and Theorem 1 as follows. First note that the set $R$ contains $N B$ rectangles, since each rectangle corresponds to an edge of the buttefly graph and each of the $N$ non-sink nodes of the butterfly graph has $B$ outgoing edges. Each of these rectangles gives one or two points in $P(G)$. Letting $n$ denote $|P(G)|$, we have $N B \leq n \leq 2 N B$. From $N=d \cdot B^{d} \leq n$ we get $d \leq \lg n$ and $d=\Theta\left(\lg _{B} N\right)$.

Given $n, w \geq \lg n$, and $S \geq n$, we now derive a lower bound on the query time. Setting $B=\frac{S}{n} w^{2}$ we have $B=\Omega\left(w^{2}\right)$ and $\lg B=\Omega\left(\lg \frac{S d}{N}\right)$ (as required by Theorem 1), where the last bound follows from $\lg \frac{S d}{N} \leq \lg \frac{S \cdot \lg n}{n / 2 B} \leq \lg \left(2 B \frac{S \cdot w}{n}\right) \leq \lg \left(2 B^{2}\right)=O(\lg B)$. Furthermore we have $\lg \frac{S w}{n}=\frac{1}{2} \lg \left(\frac{S w}{n}\right)^{2} \geq$ $\frac{1}{2} \lg \left(\frac{S}{n} w^{2}\right)=\frac{1}{2} \lg B$. From Theorem 1 we can now bound the time for a skyline counting query by $t=$ $\Omega(d)=\Omega\left(\lg _{B} N\right)=\Omega(\lg n / \lg B)=\Omega(\lg n / \lg (S w / n))$.

\section{Skyline Counting Data Structure}

In this section we describe a data structure using $O(n)$ space supporting orthogonal skyline counting queries in $O(\lg n / \lg \lg n)$ time. We first describe the basic idea of how to support queries, then present the details of the stored data structure and the details of the query.

The basic idea is to store the $n$ points in left-to-right $x$-order at the leaves of a balanced tree $T$ of degree $\Theta\left(\log ^{\varepsilon} n\right)$, i.e. height $O(\log n / \log \log n)$, and for each internal node $v$ have a list $L_{v}$ of the points in the subtree rooted in $v$ in sorted $y$-order. The slab of $v$ is the narrowest infinite vertical band containing $L_{v}$. To obtain the overall linear space bound, $L_{v}$ will not be stored explicitly but implicitly and rank-reduced using rank-select data structures, where navigation is performed using fractional cascading on rank-select data structures (details below). A 4-sided query $R$ decomposes into 2-sided subqueries at $O(\log n / \log \log n)$ nodes (in Figure 4, $R$ is decomposed into subqueries $R_{1}-R_{5}$, white points are nodes on the skyline within $R$, double circled points are the topmost points within each $R_{i}$ ). For skyline queries (both counting and reporting) it is important to consider the subqueries right-to-left, and the lower $y$-value for the subquery in $R_{i}$ is raised to the maximal $y$-value of a point in the subqueries to the right. Since the tree $T$ has nonconstant degree, we need space efficient solutions for multislab queries at each node $v$. We partition $L_{v}$ into blocks of size $O\left(\log ^{2 \varepsilon} n\right)$, and a query $R_{i}$ decomposes into five subqueries (1-5), see Figure 7: (1) and (3) are on small subsets of points within a single block and can be answered by tabulation (given the signature of the block); (2) is a block aligned multislab query; (4) and (5) are for single slabs (at the children of $v$ ). 


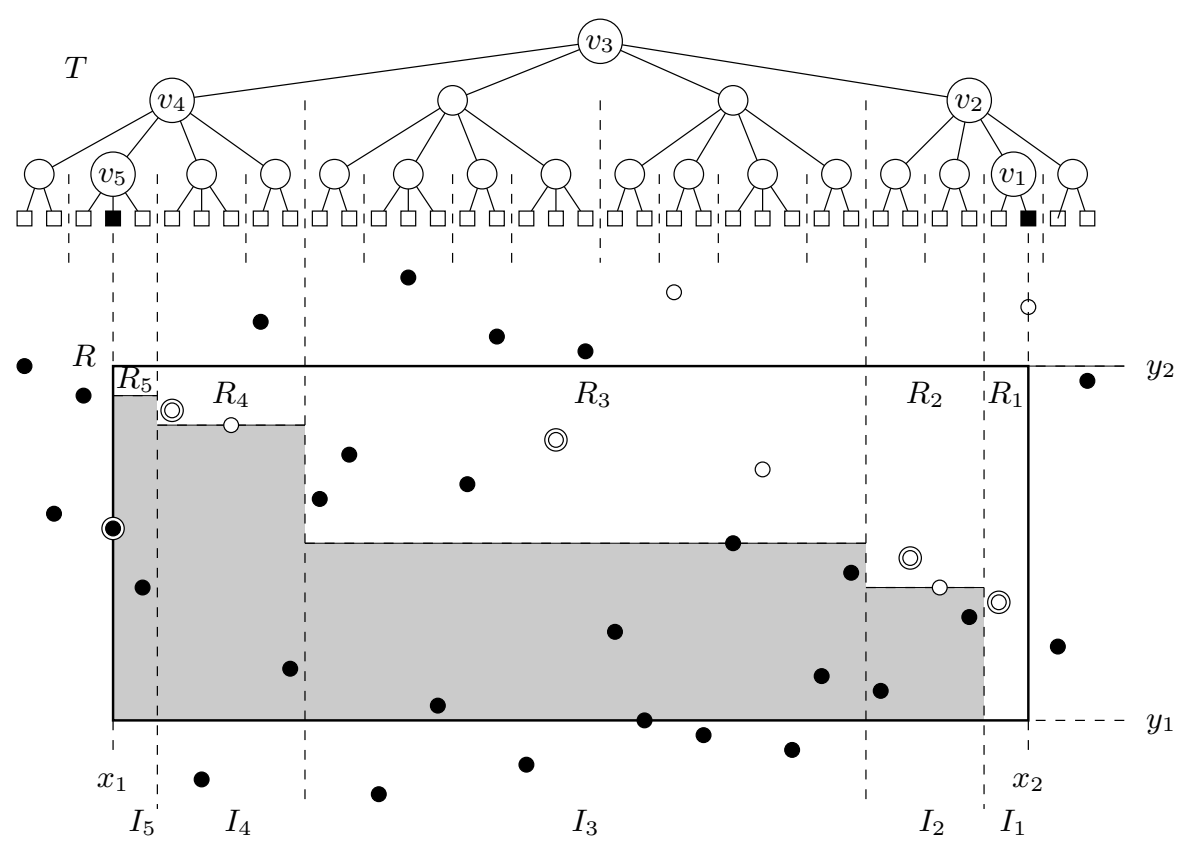

Figure 4: The base tree $T$ with $\Delta=4$, and the decomposition of a query into a sequence of multislab queries $R_{1}-R_{5}$. White points are nodes on the skyline within $R$. The double circled points are the topmost points within each of the multislabs.

For $(2,4,5)$ the skyline size between points $i$ and $j$ (numbered bottom-up) can be computed as one plus the difference between the size of the skyline from 1 to $j$ and 1 to $k$, where $k$ is the rightmost point between $i$ and $j$ (see Figure 6, white and black circles and crosses are all points, crosses indicate the skyline from $i$ to $j$, white circles from 1 to $k$, and white circles together with crosses from 1 to $j$ ). Finally, the skyline size from 1 to $i$ can be computed from a prefix sum, if we for point $i$ store the number of points in the skyline from 1 to $i-1$ dominated by $i$ (see Figure 5, the skyline between 1 and 6 consists of the three white nodes, and the size is $6-(2+0+0+0+1+0)=3)$.

We let $\Delta=\max \left\{2,\left\lceil\lg ^{\varepsilon} n\right\rceil\right\}$ be a parameter of our construction, where $0<\varepsilon<1 / 3$ is a constant. We build a balanced base tree $T$ over the set of points $P$, where the leafs from left-to-right store the points in $P$ in sorted order w.r.t. $x$-coordinate. Each internal node of $T$ has degree at most $\Delta$ and $T$ has height $\left\lceil\lg _{\Delta} n\right\rceil+1$. See Figure 4 .

For each internal node $v$ of $T$ we store a set of data structures. Before describing these we need to introduce some notation. The subtree of $T$ rooted at a node $v$ is denoted $T_{v}$, and the set of points stored at the leaves of $T_{v}$ is denoted $P_{v}$. We let $n_{v}=\left|P_{v}\right|$ and $L_{v}\left[1 . . n_{v}\right]$ be the list of the points in $P_{v}$ sorted in increasing $y$-order. We let $I_{v}=\left[\ell_{v}, r_{v}\right]$ denote the $x$-interval defined by the $x$-coordinates of the points stored at the leaves of $T_{v}$, and denote $I_{v} \times[n]$ the slab spanned by $v$. The degree of $v$ is denoted $d_{v}$, the children of $v$ are from left-to-right denoted $c_{v}^{1}, \ldots, c_{v}^{d_{v}}$, and the parent of node $v$ is denoted $p_{v}$. A list $L_{v}$ is partitioned into a sequence of blocks $B_{v}\left[1 . .\left\lceil n_{v} / \Delta^{2}\right\rceil\right]$ of size $\Delta^{2}$, such that $B_{v}[i]=L_{v}\left[(i-1) \Delta^{2}+1 . . \min \left\{n_{v}, i \Delta^{2}\right\}\right]$. The signature $\sigma_{v}[i]$ of a block $B_{v}[i]$ is a list of pairs: For each point $p$ from $B_{v}[i]$ in increasing $y$-order we construct a pair $(j, r)$, where $j$ is the index of the child $c_{v}^{j}$ of $v$ storing $p$ and $r$ is the rank of $p$ 's $x$-coordinate among all points in $B_{v}[i]$ stored at the same child $c_{v}^{j}$ as $p$. The total number of bits required for a signature is at most $\Delta^{2}\left(\lg \Delta+\lg \Delta^{2}\right)=O\left(\lg ^{2 \varepsilon} n \cdot \lg \lg n\right)$. 
To achieve overall $O(n)$ space we need to encode succinctly sufficient information for performing queries. In particular we will not store the points in $L_{v}$ explicitly at the node $v$, but only partial information about the points relative position will be stored.

Queries on a block $B_{v}[i]$ are handled using table lookups in global tables using the block signature $\sigma_{v}[i]$. We have tables for the below block queries, where we assume $\sigma$ is the signature of a block storing points $p_{1}, \ldots, p_{\Delta^{2}}$ distributed in $\Delta$ child slabs.

Below $(\sigma, t, i)$ Returns the number of points from $p_{1}, \ldots, p_{t}$ contained in slab $i$.

$\operatorname{Rightmost}(\sigma, b, t, i, j)$ Returns $k$, where $p_{k}$ is the rightmost point among $p_{b}, \ldots, p_{t}$ contained in slabs $[i, j]$. If no such point exists, -1 is returned.

Topmost $(\sigma, b, t, i, j)$ Returns $k$, where $p_{k}$ is the topmost point among $p_{b}, \ldots, p_{t}$ contained in slabs $[i, j]$. If no such point exists, -1 is returned.

SkyCount $(\sigma, b, t, i, j)$ Returns the size of the skyline for the subset of the points $p_{b}, \ldots, p_{t}$ contained in slabs $[i, j]$.

The arguments to each of the above lookups consists of at most $|\sigma|+2 \lg \Delta^{2}+2 \lg \Delta=|\sigma|+O(\lg \lg n)=$ $O\left(\lg ^{2 \varepsilon} n \cdot \lg \lg n\right)$ bits and the answer is $\lg (\Delta+1)=O(\lg \lg n)$ bits, i.e. each query can be answered in $O(1)$ time using a table of size $O\left(2^{\lg 2 \varepsilon} n \cdot \lg \lg n \cdot \lg \lg n\right)=o(n)$ bits, since $\varepsilon<1 / 3$.

For each internal node $v$ of $T$ we store the following data structures, each having $O(1)$ access time.

$C_{v}(i)$ Compact array that for each $i$, where $1 \leq i \leq n_{v}$, stores the index of the child of $v$ storing $L_{v}[i]$, i.e. $1 \leq C_{v}(i) \leq \Delta$. Space usage $O\left(n_{v} \lg \Delta\right)$ bits.

$\pi_{v}(i)$ For each $i, 1 \leq i \leq n_{v}$, stores the index of $L_{v}[i]$ in $L_{p_{v}}$, i.e. $L_{p_{v}}\left[\pi_{v}(i)\right]=L_{v}[i]$. This can be supported by constructing the select data structure of Lemma 1 on the bit-vector $X$, where $X[i]=1$ if and only if $L_{p_{v}}[i]$ is in $L_{v}$. A query to $\pi_{v}(i)$ simply becomes a select $(i)$ query. Space usage $O\left(n_{v} \lg \left(n_{p_{v}} / n_{v}\right)\right)=O\left(n_{v} \lg \Delta\right)$ bits.

$\sigma_{v}(i)$ Array of signatures for the blocks $B_{v}\left[1 . .\left\lceil n_{v} / \Delta^{2}\right\rceil\right]$. Space usage $O\left(n_{v} / \Delta^{2} \cdot \Delta^{2} \cdot \lg \Delta\right)=O\left(n_{v} \lg \Delta\right)$ bits.

$\operatorname{Pred}_{v}(t, i) / \operatorname{Succ}_{v}(t, i)$ Supports finding the predecessor/successor of $L_{v}[t]$ in the $i$ 'th child list $L_{c_{v}^{i}}$. Returns $\max \left\{k \mid 1 \leq k \leq n_{c_{v}^{i}} \wedge \pi_{c_{v}^{i}}[k] \leq t\right\}$ and $\min \left\{k \mid 1 \leq k \leq n_{c_{v}^{i}} \wedge \pi_{c_{v}^{i}}[k] \geq t\right\}$, respectively. For each child index $i$, we construct an array $X^{i}$ of size $\left\lceil n / \Delta^{2}\right\rceil$, such that $X^{i}[b]$ is the number of points in block $B_{v}[b]$ that are stored in the $i$ 'th child slab. The prefix sums of each $X^{i}$ are stored using the data structure of Lemma 2 using space $O\left(\left(n_{v} / \Delta^{2}\right) \lg \left(\Delta^{2}\right)\right)$ bits. The total space for all $\Delta$ children of $v$ becomes $O\left(\Delta \cdot n_{v} / \Delta^{2} \cdot \lg \Delta\right)=O\left(n_{v}\right)$ bits. The result of a $\operatorname{Pred}_{v}(t, i)$ query is $\sum_{j=1}^{\left\lceil t / \Delta^{2}\right\rceil-1} X^{i}[j]+\operatorname{Below}\left(\sigma_{v}\left(\left\lceil t / \Delta^{2}\right\rceil\right), 1+\left(t-1 \bmod \Delta^{2}\right), i\right)$, where the first term can be computed in $O(1)$ time by Lemma 2 and the second term is a constant time global table lookup. The result of $\operatorname{Succ}_{v}(t, i)=\operatorname{Pred}_{v}(t, i)$ if $C_{v}[t]=i$, otherwise $\operatorname{Succ}_{v}(t, i)=\operatorname{Pred}_{v}(t, i)+1$.

$\operatorname{Rightmost}_{v}(i, j)$ Returns the index $k$, where $i \leq k \leq j$, such that $L_{v}[k]$ has the maximum $x$-value among $L_{v}[i . . j]$. Using Lemma 3 on the array of the $x$-coordinates of the points in $L_{v}$ we achieve $O(1)$ time queries and space usage $O\left(n_{v}\right)$ bits. 


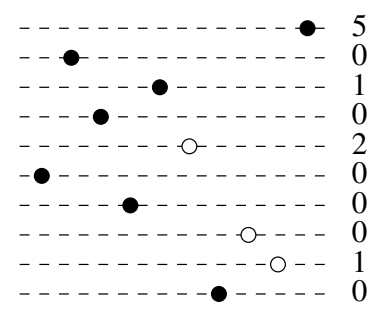

Figure 5: Computation of $\left|\operatorname{Skyline}\left(L_{v}[1 . . i]\right)\right|$. To the right of each point $L_{v}[i]$ is shown the number of points in Skyline $\left(L_{v}[1 . . i-1]\right)$ dominated by $L_{v}[i]$. The skyline of $L_{v}[1 . .6]$ consists of the three white nodes. $\mid$ Skyline $\left(L_{v}[1 . .6]\right) \mid=6-2-0-0-0-1-0=3$.

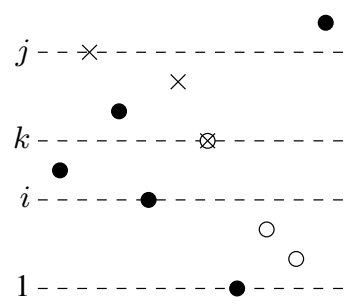

Figure 6: Illustration of $\operatorname{SkyCount}_{v}(i, j)$. White and black circles and crosses are all points. $L_{v}[k]$ is the rightmost point in $L_{v}[i . . j]$. Crosses indicate $\operatorname{Skyline}\left(L_{v}[i . . j]\right)$, white circles indicate Skyline $\left(L_{v}[1 . . k]\right)$, and white circles together with crosses is $\operatorname{Skyline}\left(L_{v}[1 . . j]\right)$.

$\operatorname{SkyCount}_{v}(i)$ Returns $\left|\operatorname{Skyline}\left(L_{v}[1 . . i]\right)\right|$. Construct an array $X$, where $X[i]$ is the number of points in Skyline $\left(L_{v}[1 . . i-1]\right)$ dominated by $L_{v}[i]$. See Figure 5 . We can now compute $\left|\operatorname{Skyline}\left(L_{v}[1 . . i]\right)\right|$ as $i-\sum_{j=1}^{i} X[j]$. Using Lemma 2 the query time becomes $O(1)$ and the space usage $O\left(n_{v}\right)$ bits, since $\sum_{j=1}^{n_{v}} X[j] \leq n_{v}-1$.

SkyCount $_{v}(i, j)$ Returns $\left|\operatorname{Skyline}\left(L_{v}[i . . j]\right)\right|$, computable by the following expression (see Figure 6):

$$
\operatorname{SkyCount}_{v}(j)-\operatorname{SkyCount}_{v}\left(\operatorname{Rightmost}_{v}(i, j)\right)+1 \text {. }
$$

Finally, we store for each node $v$ and slab interval $[i, j]$ the following data structures.

Rightmost $_{v, i, j}(b, t)$ Returns $k$, where $L_{v}[k]$ is the rightmost point among the points in blocks $B_{v}[b . . t]$ contained in slabs $[i, j]$. If no such point exists, -1 is returned. Can be solved by applying Lemma 3 to the array $X$, where $X[s]$ is the $x$-coordinate of the rightmost point in $B_{v}[s]$ contained in slabs $[i, j]$. A query first finds the block $\ell$ containing the rightmost point using this data structure, and then returns $(\ell-1) \Delta^{2}+\operatorname{Rightmost}\left(\sigma_{v}[\ell], 1, \Delta^{2}, i, j\right)$. Space usage $O\left(n_{v} / \Delta^{2}\right)$ bits.

Topmost $_{v, i, j}(b, t)$ Returns $k$, where $L_{v}[k]$ is the topmost point among the points in blocks $B_{v}[b . . t]$ contained in slabs $[i, j]$. If no such point exists, -1 is returned. Can be solved by first using Lemma 3 on the array $X$, where $X[s]=s$ if there exists a point in $B_{v}[s]$ contained in slabs $[i, j]$. Otherwise $X[s]=0$. Let $\ell$ be the block found using Lemma 3. Return the result of $(\ell-1) \Delta^{2}+\operatorname{Topmost}\left(\sigma_{v}[\ell], 1, \Delta^{2}, i, j\right)$. Space usage $O\left(n_{v} / \Delta^{2}\right)$ bits.

SkyCount $_{v, i, j}(b, t)$ Returns the size of the skyline for the subset of points in blocks $B_{v}[b . . t]$ contained in slabs $[i, j]$. Can be supported by two applications of Lemma 2 on two arrays $X$ and $Y$ as follows. 
Let $X[s]=\operatorname{SkyCount}\left(\sigma_{v}[s], 1, \Delta^{2}, i, j\right)$, i.e. the size of the skyline of the points in block $B_{v}[s]$ contained in slabs $[i, j]$. Let $B_{v, i, j}[s]$ denote the points in $B_{v}[s]$ contained in slabs $[i, j]$. Let $Y[s]=$ $\left|\operatorname{Skyline}\left(B_{v, i, j}[1 . . s-1]\right) \backslash \operatorname{Skyline}\left(B_{v, i, j}[1 . . s]\right)\right|$, i.e. the number of points on $\operatorname{Skyline}\left(B_{v, i, j}[1 . . s-1]\right)$ dominated by points in $B_{v, i, j}[s]$. Space usage for $X$ and $Y$ is $O\left(n_{v} / \Delta^{2} \cdot \lg \Delta^{2}\right)$ bits. We can compute $\operatorname{SkyCount}_{v, i, j}(b, t)=\sum_{s=k}^{t} X[s]-\sum_{s=k+1}^{t} Y[s]$, where $k=\left\lceil\operatorname{Rightmost}_{v, i, j}(b, t) / \Delta^{2}\right\rceil$.

The total space of our data structure, in addition to the $o(n)$ bits for our global tables, can be bounded as follows. The total space for all $O\left(\Delta^{2}\right)$ multislab data structures for a node $v$ is $O\left(\Delta^{2} \cdot n_{v} / \Delta^{2} \cdot \lg \Delta\right)$ bits. The total space for all data structures at a node $v$ becomes $O\left(n_{v} \lg \Delta\right)$ bits. Since the sum of all $n_{v}$ for a level of $T$ is at most $n$, the total space for all nodes at a level of $T$ is $O(n \lg \Delta)$ bits. Since $T$ has height $O\left(\lg _{\Delta} n\right)$, the total space usage becomes $O\left(n \lg \Delta \cdot \lg _{\Delta} n\right)=O(n \lg n)$ bits, i.e. $O(n)$ words. The data structure can be constructed bottom-up in $O(n \log n)$ time.

\subsection{Skyline Range Counting Queries}

To answer a skyline counting query $R=\left[x_{1}, x_{2}\right] \times\left[y_{1}, y_{2}\right]$, we identify the nodes on the paths in $T$ from the two leaves storing $x_{1}$ and $x_{2}$ up to the lowest common ancestor of the two leaves. Let $v_{1}, \ldots, v_{m}$ be the set of these nodes in a right-to-left traversal in $T$ (see Figure 4). The horizontal span of the query, $\left[x_{1}, x_{2}\right]$, is the concatenation of the span of at most one multislab $I_{1}, \ldots, I_{m}$ from each of $v_{1}, \ldots, v_{m}$. For each such multislab $I_{\ell}$ we form a new subquery $R_{\ell}=I_{\ell} \times\left[z_{\ell}, y_{2}\right]$, completely spanning the multislab in the horizontal direction and vertically has a range $\left[z_{\ell}, y_{2}\right]$, where $z_{1}=y_{1}$ and $z_{\ell}=\max \left\{z_{\ell-1}, y_{\ell}^{\max }+1\right\}$, for $\ell=2$ to $m$ and $y_{\ell}^{\max }$ is the maximal $y$-coordinate of a point in $I_{\ell-1} \times\left[1, y_{2}\right]$. By definition of the $R_{\ell}$ queries, the skyline of the points contained within $R$ is exactly the union of the skylines for each of the $R_{\ell}$ subqueries (see Figure 4), since the points in $R_{\ell}$ cannot be dominated by other points that are both in $R$ and to the right of $I_{\ell}$.

To navigate in $T$ we need to find the index of the successor of $y_{1}$ and the predecessor $y_{2}$ in each of the $L_{v_{\ell}}$ lists. We start with $y_{1}$ and $y_{2}$ being the indexes at the root, and then use the $\operatorname{Succ}_{v} / \operatorname{Pred}_{v}$ structures at the nodes to find the successor of $y_{1}$ and predecessor of $y_{2}$ at all the nodes on the two paths from the root to $x_{1}$ and $x_{2}$. To find the topmost point below $y_{2}$ in a multislab we use Topmost ${ }_{v, i, j}$. To navigate $y^{\max }$ values up and down between the levels of $T$ we use $\pi_{v}\left(y^{\max }\right)$ to move upwards and $\operatorname{Succ}_{v}\left(y^{\max }, j\right)$ to move downwards to a slab $j$. These navigations can be performed in $O(1)$ time per node on the paths, i.e. total time $O\left(\lg _{\Delta} n\right)$.

What remains is to compute in $O(1)$ time the size the skyline within a query range $R_{\ell}$. In the following we consider a query range that horizontally spans the child slabs $[i, j]$ of a node $v$, and vertically spans the indexes $\left[y_{\text {bottom }}, y_{\text {top }}\right]$ in $L_{v}$.

If the query range is within a single block of $L_{v}$ (i.e. $\left\lceil y_{\text {bottom }} / \Delta^{2}\right\rceil=\left\lceil y_{\text {top }} / \Delta^{2}\right\rceil$ ), we compute the skyline size as

$$
\operatorname{SkyCount}\left(\sigma_{v}\left(\left\lceil y_{\text {top }} / \Delta^{2}\right\rceil\right), 1+\left(y_{\text {bottom }}-1 \bmod \Delta^{2}\right), 1+\left(y_{\text {top }}-1 \bmod \Delta^{2}\right), i, j\right) .
$$

Otherwise we decompose the skyline counting query into five subranges (1)-(5), see Figure 7. We first compute the $y$-coordinate of the rightmost point $p_{1}$ in the top block $B_{\text {top }}$ of the query range using

$$
p_{1} . y=\operatorname{Rightmost}\left(\sigma_{v}\left(\left\lceil y_{\text {top }} / \Delta^{2}\right\rceil\right), 1,1+\left(y_{\text {top }}-1 \bmod \Delta^{2}\right), i, j\right)+\Delta^{2}\left\lceil y_{\text {top }} / \Delta^{2}-1\right\rceil,
$$

and compute the size of the skyline of the intersection of $B_{\text {top }}$ and the query region by:

$$
\operatorname{SkyCount}\left(\sigma_{v}\left(\left\lceil y_{\text {top }} / \Delta^{2}\right\rceil\right), 1,1+\left(y_{\text {top }}-1 \bmod \Delta^{2}\right), i, j\right) \text {. }
$$




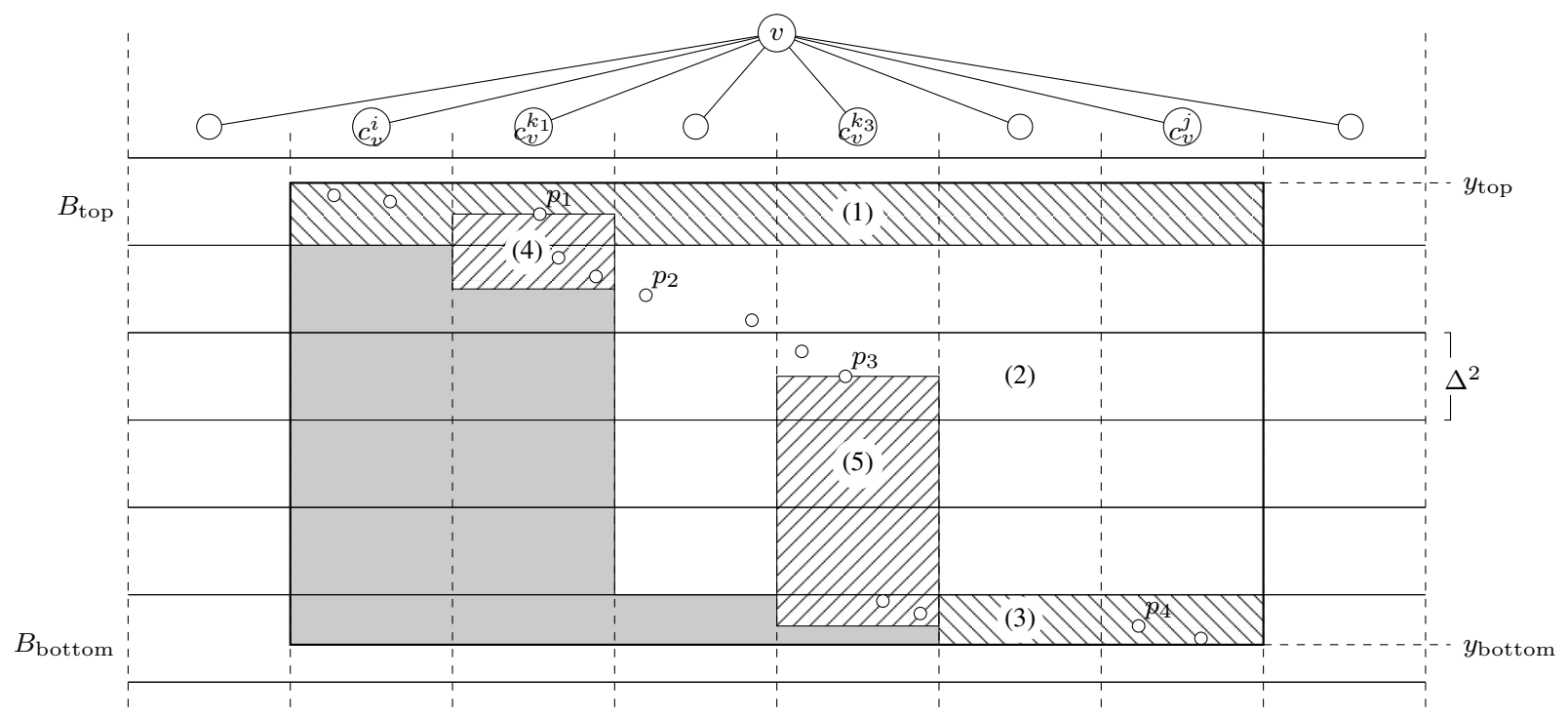

Figure 7: Skyline queries for multislabs.

Let $k_{1}$ be the slab containing $p_{1}$, computable as $k_{1}=C_{v}\left(p_{1} . y\right)$. If no point is found in block $B_{\text {top }}$, then $k_{1}=i-1$.

Next we compute the $y$-coordinate of the topmost point $p_{2}$ in the multislab query range spanning slabs $\left[k_{1}+1, j\right]$ and all blocks between $B_{\text {bottom }}$ and $B_{\text {top }}$.

$$
p_{2} \cdot y=\text { Topmost }_{v, k_{1}+1, j}\left(\left\lceil y_{\text {bottom }} / \Delta^{2}\right\rceil+1,\left\lceil y_{\text {top }} / \Delta^{2}\right\rceil-1\right) .
$$

In the same subrange we find the $y$-coordinate of the rightmost point $p_{3}$ using

$$
p_{3} . y=\operatorname{Rightmost}_{v, k_{1}+1, j}\left(\left\lceil y_{\mathrm{bottom}} / \Delta^{2}\right\rceil+1,\left\lceil y_{\mathrm{top}} / \Delta^{2}\right\rceil-1\right) .
$$

Finally, the number of points on the skyline between $p_{2}$ and $p_{3}$ (including $p_{2}$ and $p_{3}$ ) is computed by

$$
\text { SkyCount }_{v, k_{1}+1, j}\left(\left\lceil y_{\text {bottom }} / \Delta^{2}\right\rceil+1,\left\lceil y_{\text {top }} / \Delta^{2}\right\rceil-1\right) \text {. }
$$

The slab containing the point $p_{3}$ is $k_{3}=C_{v}\left(p_{3} . y\right)$. We compute the number of points on the skyline to the right of $p_{3}$ in block $B_{\text {bottom }}$ by

$$
\operatorname{SkyCount}\left(\sigma_{v}\left(\left\lceil y_{\text {bottom }} / \Delta^{2}\right\rceil\right), 1+\left(y_{\text {bottom }}-1 \bmod \Delta^{2}\right), \Delta^{2}, k_{3}+1, j\right),
$$

and the $y$-coordinate of the topmost point $p_{4}$ in block $B_{\text {bottom }}$ contained in slabs $\left[k_{3}+1, j\right]$ by $p_{4} . y=\operatorname{Topmost}\left(\sigma_{v}\left(\left\lceil y_{\text {bottom }} / \Delta^{2}\right\rceil\right), 1+\left(y_{\text {bottom }}-1 \bmod \Delta^{2}\right), \Delta^{2}, k_{3}+1, j\right)+\Delta^{2}\left\lceil y_{\text {bottom }} / \Delta^{2}-1\right\rceil$.

The remaining points to be counted are the skyline points in slab $k_{1}$ between $p_{1}$ and $p_{2}$, and in slab $k_{3}$ between $p_{3}$ and the point $p_{4}$ in block $B_{\text {bottom. These values can be computed by }}$

$$
\begin{gathered}
\operatorname{SkyCount}_{c_{v} k_{1}}\left(\operatorname{Succ}_{v}\left(p_{2} \cdot y+1, k_{1}\right), \operatorname{Pred}_{v}\left(p_{1} \cdot y, k_{1}\right)\right)-1 \\
\operatorname{SkyCount}_{c_{v}}\left(\operatorname{Succ}_{v}\left(p_{4} \cdot y, k_{3}\right), \operatorname{Pred}_{v}\left(p_{3} \cdot y, k_{3}\right)\right)-1,
\end{gathered}
$$


where we subtract one in both expressions, to avoid double counting $p_{1}$ and $p_{3}$.

Figure 7 illustrates the five partial counts computed. In the above we assumed that all queries ranges were non-empty. In case $p_{1}$ does not exist, then $k_{1}=i-1$ and (4) is not computed. If $p_{4}$ does not exist, then (5) stretches down to $y_{\text {bottom. If }} p_{2}$ and $p_{3}$ do not exist ( $p_{2}$ and $p_{3}$ are the same point if (4) only contains one maximal point), then (2) and (5) are not computed, the leftmost slab of (3) is $k_{1}+1$, and (4) stretches down to $p_{4} . y+1$.

To summarize, it follows that the skyline size for each multislab query $R_{\ell}$ can be computed in $O(1)$ time, and the total time for a skyline counting query becomes $O(\lg n / \lg \lg n)$.

\section{Skyline Range Reporting}

In this section, we show how to extend our skyline range counting data structure from Section 3 to also support reporting. Given a query rectangle $R=\left[x_{1}, x_{2}\right] \times\left[y_{1}, y_{2}\right]$, we let $v_{1}, \ldots, v_{m}$ and $I_{1}, \ldots, I_{m}$ be defined as in Section 3.1. The goal is to report the skyline for each of the subqueries $R_{\ell}=I_{\ell} \times\left[z_{\ell}, y_{2}\right]$ where $z_{1}=y_{1}$ and $z_{\ell}=\max \left\{z_{\ell-1}, y_{\ell}^{\max }+1\right\}$ for $\ell=2$ to $m$ and $y_{\ell}^{\max }$ is the maximal $y$-coordinate of a point in $I_{1} \times\left[1, y_{2}\right]$. Using the approach from Section 3.1 we assume the $z_{\ell}$ 's have been computed as well as the index of the successor of $y_{1}$ and the index of the predecessor of $z_{\ell}$ in each of the $L_{v_{\ell}}$ lists. Recall the lists $L_{v}$ are not stored explicitly.

To answer the query $R_{\ell}$ at a node $v=v_{\ell}$, let $[i, j]$ be the range of children of $v$ that are spanned by $R_{\ell}$ in the horizontal direction and let $y_{\text {bottom }}$ be the index of the successor of $z_{\ell}$ in $L_{v}$ and $y_{\text {top }}$ the index of the predecessor of $y_{2}$ in $L_{v}$. We first produce an output list $Y_{\ell}$ storing each point of $\operatorname{Skyline}\left(R_{\ell} \cap P_{v}\right)$ as an index into $L_{v}$. The key observation for producing this list is that the skyline inside a query rectangle is the set of points produced by the following procedure: First report the rightmost point in the query range and then recurse on the query rectangle obtained by moving the bottom side of the query to just above the returned point.

We implement this strategy in the following. First, if $R_{\ell}$ is completely within one block (block $\left\lceil y_{\text {top }} / \Delta^{2}\right\rceil$ of $L_{v}$ ), we answer it by first running

$$
\operatorname{Rightmost}\left(\sigma_{v}\left(\left\lceil y_{\text {top }} / \Delta^{2}\right\rceil\right), 1+\left(y_{\text {bottom }}-1 \bmod \Delta^{2}\right), 1+\left(y_{\text {top }}-1 \bmod \Delta^{2}\right), i, j\right) .
$$

Adding $\Delta^{2}\left\lceil y_{\text {top }} / \Delta^{2}-1\right\rceil$ to the returned value gives the index $k$ into $L_{v}$ of the rightmost point in the output. We add $k$ to $Y_{\ell}$ and recurse on the query rectangle with $y$-range from $k+1$ to $y_{\text {top }}$.

If the query range is not contained in one block, we use the decomposition into five queries that was introduced in Section 3.1, see Figure 7. We define $p_{1}, \ldots, p_{4}$ and $(1), \ldots,(5)$ as in Section 3.1. The subquery (1) is answered as just described for the case of a query range completely within a block. The query $(4)$ is answered using first the query Rightmost ${ }_{c_{v}}{ }_{k_{1}}\left(\operatorname{Succ}_{v}\left(p_{2} . y+1, k_{1}\right), \operatorname{Pred}_{v}\left(p_{1} . y, k_{1}\right)\right)$. Following that, we move the bottom of the query rectangle just above the returned point and recurse.

The query range (2) is answered by first repeatedly using the Rightmost ${ }_{v, k_{1}+1, j}$ operation to identify the blocks within the query range (2) containing points from Skyline $\left(R_{\ell} \cap P_{v}\right)$. Let $q_{1}, \ldots, q_{t}$ be the indexes into $L_{v}$ of the rightmost points returned in each of these blocks, computable by

$$
q_{1}=\text { Rightmost }_{v, k_{1}+1, j}\left(\left\lceil y_{\text {bottom }} / \Delta^{2}\right\rceil+1,\left\lceil y_{\text {top }} / \Delta^{2}\right\rceil-1\right),
$$

and for $r>1$ (until no further point is found) by

$$
q_{r}=\operatorname{Rightmost}_{v, k_{1}+1, j}\left(\left\lceil q_{r-1} / \Delta^{2}\right\rceil+1,\left\lceil y_{\text {top }} / \Delta^{2}\right\rceil-1\right) .
$$


Within each block $\left\lceil q_{r} / \Delta^{2}\right\rceil$ we compute the additional points that should be reported within slabs $\left[k_{1}, j\right]$ from right-to-left, starting with

$$
\operatorname{Rightmost}\left(\sigma_{v}\left(\left\lceil q_{r} / \Delta^{2}\right\rceil\right), 2+\left(q_{r}-1 \bmod \Delta^{2}\right), \Delta^{2}, k_{1}, j\right),
$$

until no point is found or we find the first point $f$ that should not be reported, i.e. $f$ is dominated by $q_{r+1}$, which can be checked by the condition $\gamma<C_{v}\left(q_{r+1}\right)$ or $\gamma=C_{v}\left(q_{r+1}\right)$ and $q^{\prime}=$ Rightmost $_{v^{\prime}}\left(f^{\prime}, q^{\prime}\right)$, where $\gamma=C_{v}(f), v^{\prime}=c_{v}^{\gamma}$ and $f^{\prime}=\operatorname{Pred}_{v}(f, \gamma)$, and $q^{\prime}=\operatorname{Pred}_{v}\left(q_{r+1}, \gamma\right)$.

The query (5) is answered by repeatedly using Rightmost ${ }_{c_{v}}{ }_{3}$ and finally we answer (3) using

$$
\operatorname{Rightmost}\left(\sigma_{v}\left(\left\lceil y_{\text {bottom }} / \Delta^{2}\right\rceil\right), 1+\left(y_{\text {bottom }}-1 \bmod \Delta^{2}\right), \Delta^{2}, k_{3}+1, j\right)
$$

and recursing above the returned point. It follows that the list $Y_{\ell}$ is produced in $O\left(1+\left|\operatorname{Skyline}\left(R_{\ell} \cap P_{v}\right)\right|\right)=$ $O\left(1+\left|\operatorname{Skyline}\left(R_{\ell} \cap P\right)\right|\right)$ time. Summing over all lists $Y_{\ell}$, we get a total time of $O(\lg n / \lg \lg n+k)$.

What remains is to map the indices in the lists $Y_{\ell}$ to the actual coordinates of the corresponding points. Using the $\pi$ arrays, this can be done by repeatedly determining the position of $L_{v}[i]$ in $L_{p_{v}}$. Doing this for all $O(\lg n / \lg \lg n)$ levels of the tree allows one to deduce the global $y$-rank of the point corresponding to $L_{v}[i]$. Storing an additional $O(n)$ sized array mapping global $y$-ranks to the corresponding points gives a total running time of $O((1+k) \lg n / \lg \lg n)$. To speed this up, we use the Ball-Inheritance structure of [4]. For completeness, we describe how this data structure is implemented in terms of the $\pi$ arrays we have defined: Let $B \geq 2$ be a parameter. For every level $j$ in the base tree $T$ that is a multiple of $B^{i}$, for $i=0, \ldots, \lg _{B} \lg _{\Delta} n$, we let all nodes $v$ at level $j$ store the following array:

$\pi_{v}^{\left(B^{i}\right)}(j)$ For each $j, 1 \leq j \leq n_{v}$, stores the index of $L_{v}[j]$ in $L_{u(v)}$. Here $u(v)$ is the ancestor of $v$ at the nearest level that is a multiple of $B^{i+1}$ (excluding possibly the level storing $v$ ). This can be supported by constructing the select data structure of Lemma 1 on the bit-vector $X$, where $X[j]=1$ if and only if $L_{u(v)}[j]$ is in $L_{v}$. A query $\pi_{v}^{\left(B^{i}\right)}(j)$ becomes select $(j)$. The space usage for $\pi_{v}^{\left(B^{i}\right)}$ becomes $O\left(n_{v} \lg \left(n_{u(v)} / n_{v}\right)\right)=O\left(n_{v} \lg \left(\Delta^{B^{i+1}}\right)\right)=O\left(n_{v} B^{i+1} \lg \Delta\right)$ bits.

Given an index $i$ into $L_{v}$, we can now recover $L_{v}[i]$ by using the $\pi$ arrays to first jump $B$ levels up, then $B^{2}$ levels up and so forth. The number of jumps becomes $O\left(\lg _{B} \lg _{\Delta} n\right)$ and hence we get a query time of $O\left(\lg n / \lg \lg n+k \lg _{B} \lg _{\Delta} n\right)$. The total space usage for all $\pi$ arrays becomes

$$
O\left(\sum_{i=1}^{\lg _{B} \lg _{\Delta} n} \frac{\lg _{\Delta} n}{B^{i}} \cdot B^{i+1} \lg \Delta\right)=O\left(n \lg n \cdot\left(B \lg _{B} \lg _{\Delta} n\right)\right)
$$

bits. Setting $B=\lg ^{\varepsilon} n$ for an arbitrarily small constant $\varepsilon>0$ gives a data structure with query time $O(\lg n / \lg \lg n+k)$ and space usage $O\left(n \lg ^{\varepsilon} n\right)$ words. Setting $B=2$ gives a data structure with query time $O(\lg n / \lg \lg n+k \lg \lg n)$ and space usage $O(n \lg \lg n)$ words as claimed.

\section{References}

[1] Stephen Alstrup, Gerth Stølting Brodal, and Theis Rauhe. New data structures for orthogonal range searching. In 41st Annual Symposium on Foundations of Computer Science, pages 198-207. IEEE Computer Society, 2000. 
[2] Paul Beame and Faith E. Fich. Optimal bounds for the predecessor problem. In 31st Annual ACM Symposium on Theory of Computing, pages 295-304. ACM, 1999.

[3] Gerth Stølting Brodal and Konstantinos Tsakalidis. Dynamic planar range maxima queries. In 38th International Colloquium on Automata, Languages, and Programming, volume 6755 of Lecture Notes in Computer Science, pages 256-267. Springer Verlag, Berlin, 2011.

[4] Timothy M. Chan, Kasper Green Larsen, and Mihai Pătraşcu. Orthogonal range searching on the RAM, revisited. In 27th ACM Symposium on Computational Geometry, pages 1-10. ACM, 2011.

[5] Ananda Swarup Das, Prosenjit Gupta, Anil Kishore Kalavagattu, Jatin Agarwal, Kannan Srinathan, and Kishore Kothapalli. Range aggregate maximal points in the plane. In 6th International Workshop Algorithms and Computation, volume 7157 of Lecture Notes in Computer Science, pages 52-63. Springer, 2012.

[6] Ananda Swarup Das, Prosenjit Gupta, and Kannan Srinathan. Counting maximal points in a query orthogonal rectangle. In 7th International Workshop on Algorithms and Computation, volume 7748 of LNCS, pages 65-76. Springer, 2013.

[7] Johannes Fischer. Optimal succinctness for range minimum queries. In 9th Latin American Symposium on Theoretical Informatics, volume 6034 of Lecture Notes in Computer Science, pages 158-169. Springer, 2010.

[8] Joseph JáJá, Christian Worm Mortensen, and Qingmin Shi. Space-efficient and fast algorithms for multidimensional dominance reporting and counting. In 15th International Symposium Algorithms and Computation, volume 3341 of Lecture Notes in Computer Science, pages 558-568. Springer, 2004.

[9] Anil Kishore Kalavagattu, Jatin Agarwal, Ananda Swarup Das, and Kishore Kothapalli. Counting range maxima points in plane. In International Workshop On Combinatorial Algorithms, volume 7643 of Lecture Notes in Computer Science, pages 263-273. Springer, 2012.

[10] Anil Kishore Kalavagattu, Ananda Swarup Das, Kishore Kothapalli, and Kannan Srinathan. On finding skyline points for range queries in plane. In 23rd Annual Canadian Conference on Computational Geometry, 2011.

[11] Yakov Nekrich and Gonzalo Navarro. Sorted range reporting. In 13th Scandinavian Symposium and Workshops Algorithm Theory, volume 7357 of Lecture Notes in Computer Science, pages 271-282. Springer, 2012.

[12] Mihai Pătraşcu. Unifying the landscape of cell-probe lower bounds. SIAM Journal on Computing, 40(3):827-847, 2011.

[13] Mihai Pătraşcu. Lower bounds for 2-dimensional range counting. In 39th Annual ACM Symposium on Theory of Computing, pages 40-46. ACM, 2007.

[14] Mihai Pătraşcu and Mikkel Thorup. Time-space trade-offs for predecessor search. In 38th Annual ACM Symposium on Theory of Computing, pages 232-240. ACM, 2006.

[15] Rajeev Raman, Venkatesh Raman, and S. Srinivasa Rao. Succinct indexable dictionaries with applications to encoding $k$-ary trees and multisets. In 13th Annual ACM-SIAM Symposium on Discrete Algorithms, pages 233-242. SIAM, 2002.

[16] Rajeev Raman, Venkatesh Raman, and Srinivasa Rao Satti. Succinct indexable dictionaries with applications to encoding $k$-ary trees, prefix sums and multisets. ACM Transactions on Algorithms, 3(4), 2007.

[17] Kunihiko Sadakane. Succinct data structures for flexible text retrieval systems. Journal of Discrete Algorithms, 5(1):12-22, 2007.

[18] Andrew Chi Chih Yao. Should tables be sorted? Journal of the ACM, 28(3):615-628, 1981. 